\title{
THE DUAL OF A BERGMAN SPACE ON SIMPLY CONNECTED DOMAINS
}

By

HÅKAN HEDENMALM

In memory of Tom Wolff

\section{Introduction}

The Bergman spaces and duality. Let $\Omega$ be a domain (open connected subset) in the complex plane. For $0<p<+\infty$, the Bergman p-space, or Bergman space of exponent $p$, denoted $A^{p}(\Omega)$, consists of all holomorphic functions $f$ on $\Omega$ subject to the norm finiteness condition

$$
\|f\|_{A^{p}(\Omega)}=\left(\int_{\Omega}|f(z)|^{p} d A(z)\right)^{1 / p}<+\infty,
$$

where $d A(z)=\pi^{-1} d x d y$ (with $z=x+i y$ ) is normalized area measure in the plane. For $1 \leq p<+\infty$, this defines a norm, and hence $A^{p}(\Omega)$ is a Banach space in this range of $p$; for $0<p<1$, we have a quasi-norm, which makes $A^{p}(\Omega)$ a quasiBanach space. Of course, this space degenerates if the boundary of $\Omega$ is too thin; for instance, if $\Omega=\mathbb{C}$, then $A^{p}(\Omega)$ consists only of the zero function. For $p=2$, the space $A^{2}(\Omega)$ is non-trivial if and only if the complement $\mathbb{C} \backslash \Omega$ has positive logarithmic capacity [15]; for $2<p<+\infty$, only partial results are known [15] (the reason probably is that as $p \rightarrow+\infty$, we get $H^{\infty}(\Omega)$ as limit space, and a capacitytype criterion for finite $p$ should converge to one involving analytic capacity, a quantity that seems hard to describe in geometric-analytic terms). We mention in passing that we have the following dichotomy: either $A^{2}(\Omega)$ is trivial, or it is infinite-dimensional [41]. The proof in [41] carries over to $A^{p}(\Omega)$ for $2 \leq p<+\infty$; however, for smaller $p$, this is false, as is seen from the example $A^{p}(\mathbb{C} \backslash\{0,1\})$, which is one-dimensional, being spanned by the function $1 /(z(z-1))$, for $1<p<2$. For $p=2$, we have a Hilbert space which, as such, is self-dual:

$$
A^{2}(\Omega)^{*}=A^{2}(\Omega) .
$$


We now restrict $p$ to the interval $1<p<+\infty$, and let $p^{\prime}$ be the dual exponent to $p$ defined by

$$
1 / p+1 / p^{\prime}=1
$$

The question arises as to whether we can represent the dual space of $A^{p}(\Omega)$ as $A^{p^{\prime}}(\Omega)$ with respect to the dual action induced by $A^{2}(\Omega)$ :

$$
\langle f, g\rangle=\int_{\Omega} f(z) \bar{g}(z) d A(z), \quad f \in A^{\mathcal{p}}(\Omega), g \in A^{p^{\prime}}(\Omega) .
$$

The example $\Omega=\mathbb{C} \backslash\{0,1\}$ shows that some kind of geometric requirement on $\Omega$ is needed to have the duality, unless $p=2$. Clearly, any function $g \in A^{p^{\prime}}(\Omega)$ gives rise to a continuous linear functional. However, it is not immediately clear that $g \neq 0$ guarantees that the induced functional is nontrivial. In fact, for the exterior disk $\Omega=\{z \in \mathbb{C}:|z|>1\}$, the function $f(z)=1 / z$ belongs to $A^{p}(\Omega)$ for all $p$ with $2<p<+\infty$, whereas it is easily checked that $\langle f, g\rangle_{\Omega}=0$ for all $g \in A^{p^{\prime}}(\Omega)$ in that range of $p$. For the converse direction, we apply the Hahn-Banach theorem to represent the dual elements as functions in $L^{p^{\prime}}(\Omega)$, and obtain the identification

$$
A^{p}(\Omega)^{*}=L^{p^{\prime}}(\Omega) / A^{p}(\Omega)^{\perp}
$$

where $A^{p}(\Omega)^{\perp}$ denotes the annihilator of $A^{p}(\Omega)$ in $L^{p^{\prime}}(\Omega)$. We realize that the hypothetical identification

$$
A^{p}(\Omega)^{*}=A^{p^{\prime}}(\Omega)
$$

requires two things:

$$
A^{p^{\prime}}(\Omega) \cap A^{p}(\Omega)^{\perp}=\{0\}, \quad A^{p^{\prime}}(\Omega)+A^{p}(\Omega)^{\perp}=L^{p^{\prime}}(\Omega) .
$$

In view of basic functional analysis, we ask for a reverse triangle inequality:

$$
\varepsilon\left(\|f\|_{L^{p^{\prime}}(\Omega)}+\|g\|_{L^{p^{\prime}}(\Omega)}\right) \leq\|f+g\|_{L^{p^{\prime}}(\Omega)}, \quad f \in A^{p^{\prime}}(\Omega), g \in A^{p}(\Omega)^{\perp}
$$

for some positive constant $\varepsilon$. But we also need to know that $A^{p^{\prime}}(\Omega)+A^{p}(\Omega)^{\perp}$ is dense in $L^{p^{\prime}}(\Omega)$. By duality, it is sufficient to consider the case $p>2$. If $\Omega$ has finite area, we have the inclusions

$$
A^{2}(\Omega) \subset A^{p^{\prime}}(\Omega), \quad A^{2}(\Omega)^{\perp} \subset A^{p}(\Omega)^{\perp},
$$

which lead to

$$
L^{2}(\Omega)=A^{2}(\Omega)+A^{2}(\Omega)^{\perp} \subset A^{p^{\prime}}(\Omega)+A^{p}(\Omega)^{\perp} ;
$$

also, clearly, $L^{2}(\Omega)$ is dense in $L^{p^{\prime}}(\Omega)$, which does it. 
The boundedness of the Bergman projection. Taking (1.3) into account, we realize that (1.2) requires the existence of a continuous projection $\Pi_{p^{\prime}}: L^{p^{\prime}}(\Omega) \rightarrow A^{p^{\prime}}(\Omega)$ that is onto and has $A^{p}(\Omega)^{\perp}$ as kernel. For $p=2$, this projection is effected by the orthogonal projection $\Pi_{2}: L^{2}(\Omega) \rightarrow A^{2}(\Omega)$, usually referred to as the Bergman projection. The Bergman projection can be represented in terms of an integral kernel $K_{\Omega}$, known as the Bergman kernel:

$$
\Pi_{2} f(z)=\int_{\Omega} K_{\Omega}(z, \zeta) f(\zeta) d A(\zeta), \quad z \in \Omega
$$

for $f \in L^{2}(\Omega)$. If we think of $\Pi_{p^{\prime}}$ as a linear mapping $L^{p^{\prime}}(\Omega) \rightarrow L^{p^{\prime}}(\Omega)$, it has an adjoint $\Pi_{p^{\prime}}^{*}: L^{p}(\Omega) \rightarrow L^{p}(\Omega)$, which then is a projection onto $A^{p}(\Omega)$ with kernel $A^{p^{\prime}}(\Omega)^{\perp}$. This means that if we have found $\Pi_{p^{\prime}}$, we automatically also have $\Pi_{p}=\Pi_{p^{\prime}}^{*}$. We need to identify the projection $\Pi_{p^{\prime}}$. If $p>2$ (that is, $p^{\prime}<2$ ), it follows from the above comparison between $L^{p^{\prime}}(\Omega)$ and $L^{2}(\Omega)$ that at least if $\Omega$ has finite area, the sought-after $\Pi_{p^{\prime}}$ (and hence also $\Pi_{p}$ ) must coincide with the Bergman projection on $L^{2}(\Omega)$. This means that our question takes the following form:

- For which $\Omega$ and $p$ does the Bergman projection $\Pi=\Pi_{2}$ extend continuously to an operator $L^{p}(\Omega) \rightarrow A^{p}(\Omega)$ ?

In Sections 2 and 3, we consider the problem of to what extent this reformulation is equivalent to the duality problem also in case the underlying domain has infinite area.

The kernel $K_{\Omega}$ can be expressed as

$$
K_{\Omega}(z, \zeta)=\sum_{n=1}^{+\infty} e_{n}(z) \bar{e}_{n}(\zeta), \quad(z, \zeta) \in \Omega \times \Omega,
$$

where $e_{1}, e_{2}, e_{3}, \ldots$ is some orthonormal basis in $A^{2}(\Omega)$. As the kernel is uniquely determined by the reproducing property

$$
f(z)=\Pi f(z)=\left\langle f, K_{\Omega}(\cdot, z)\right\rangle_{\Omega}=\int_{\Omega} K_{\Omega}(z, \zeta) f(\zeta) d A(\zeta), \quad z \in \Omega,
$$

for $f \in A^{2}(\Omega)$, the choice of orthogonal basis is unimportant in the above series representation.

The reformulated problem above (in terms of the boundedness of the Bergman projection) has been studied by Burbea [13] and Solovyov [37, 38], and later, by Békollé [6]. Solovyov shows that, for a class of domains, the boundedness of the Bergman projection on $L^{p}$ can be described in terms of geometric properties of the boundary, which are automatically fulfilled for $\frac{4}{3}<p<4$. Burbea, on the 
other hand, uses the universal covering map to lift the general problem to the unit disk in a weighted setting, and obtains a characterization of whether the Bergman projection is bounded in terms of Muckenhoupt $\left(A_{p}\right)$ conditions along zig-zag loops.

None of the above results can be reasonably used to decide whether the Bergman projection is bounded on $L^{p}$ for all simply connected domains and all $p$ in some nonempty open interval around 2 . This, then, is our main result:

Theorem 1.1. Let $\Omega$ be a simply connected domain in $\mathbb{C}$, other than the plane itself. There exists a universal constant $p_{0}, \frac{4}{3} \leq p_{0}<2$, independent of $\Omega$, such that the Bergman projection $\Pi: L^{2}(\Omega) \rightarrow A^{2}(\Omega)$ extends to a bounded projection $L^{p}(\Omega) \rightarrow A^{p}(\Omega)$ for all $p$ in the interval $p_{0}<p<p_{0}^{\prime}$, where $p_{0}^{\prime}$ is the dual exponent: $1 / p_{0}+1 / p_{0}^{\prime}=1$. Moreover, the boundedness of the Bergman projection fails in general outside the closed interval $\left[p_{0}, p_{0}^{\prime}\right]$.

We should point out that a remark made by Békollé $[6$, p. 136] goes in the same general direction.

It is a consequence of the above theorem that $A^{p}(\Omega)^{*}=A^{p^{\prime}}(\Omega)$ holds for all $p$ with $p_{0}<p<p_{0}^{\prime}$ and all simply connected domains. Moreover, this identification of the dual space fails generally outside the interval $\left[p_{0}, p_{0}^{\prime}\right]$. The reason why the range of $p$ for which the assertion of the theorem holds is an interval of the type ]$p_{0}, p_{0}^{\prime}[$ is two-fold:

- if $\Pi$ defines a bounded projection $L^{p}(\Omega) \rightarrow A^{p}(\Omega)$, then - by duality - it also acts boundedly $L^{p^{\prime}}(\Omega) \rightarrow A^{p^{\prime}}(\Omega)$,

- if $\Pi$ defines a bounded projection $L^{p}(\Omega) \rightarrow A^{p}(\Omega)$ for some $p=p_{1}, 1<p_{1}<2$, then it does so for the intermediate parameter values $p, p_{1}<p<2$, by the RieszThorin interpolation theorem (see any book on interpolation theory).

It should be pointed out that the exact value of the constant $p_{0}$ is not known; however, the estimate $p_{0} \leq p_{1} \approx 1.413$ is obtained in Section 3 . We shall see that the statement $p_{0}=\frac{4}{3}$ is equivalent to a famous conjecture due to James Brennan [14]. It is easy to see that $p_{0}$ cannot be smaller than $\frac{4}{3}$; the configuration of $\Omega$ that does this has a "needle" in the boundary pointing into the domain. If we restrict our attention to Lipschitz domains, the value of the corresponding constant $p_{0}$ is indeed known to be $\frac{4}{3}$. This can be deduced from $[19,29]$; also, some related results are obtained for NTA domains in [34].

The assumption that $\Omega$ be simply connected cannot be omitted in the theorem, because - as we shall see later toward the end of the paper - the assertion of the theorem corresponds to certain behavior of the Green function which can be made to fail for general (non-simply connected) domains. 
The Dirichlet problem on simply connected domains. The above main result can be formulated in terms of the solvability of the Dirichlet problem. To simplify the exposition, we consider bounded domains $\Omega$ only. Let $p$ be in the interval $1<p<+\infty$. The Sobolev space $W^{1, p}(\mathbb{C})$ consists of all functions in $L^{p}(\mathbb{C})$ whose first order partial derivatives (in the sense of distribution theory) are also in $L^{p}(\mathbb{C})$; it is a Banach space when equipped with the norm

$$
\|f\|_{W^{1, p}(\mathbb{C})}=\left(\|f\|_{L^{p}(\mathbb{C})}^{p}+\left\|\partial_{z} f\right\|_{L^{p}(\mathbb{C})}^{p}+\left\|\bar{\partial}_{z} f\right\|_{L^{p}(\mathbb{C})}^{p}\right)^{1 / p}
$$

where $\partial_{z}, \bar{\partial}_{z}$ are the standard Wirtinger derivatives (see below). We define the Sobolev space $W^{1, p}(\Omega)$ as the space of restrictions to $\Omega$ of functions in $W^{1, p}(\mathbb{C})$. It, too, is a Banach space, the norm being defined as the infimum of the norms of the various extensions to $W^{1, p}(\mathbb{C})$ of the given function. We also need the space $W_{0}^{1, p}(\Omega)$, which is defined as the closure of $C_{c}^{\infty}(\Omega)-$ the $C^{\infty}$-smooth functions on $\mathbb{C}$ whose supports are contained in $\Omega$ - in the space $W^{1, p}(\mathbb{C})$. The functions in $W_{0}^{1, p}(\Omega)$ then turn out to be determined uniquely by their values on $\Omega$ (in fact, they vanish off $\Omega$; see Section 4 ), enabling us to view $W_{0}^{1, p}(\Omega)$ as a closed subspace of $W^{1, p}(\Omega)$. We should think of $W_{0}^{1, p}(\Omega)$ as consisting of those functions in $W^{1, p}(\Omega)$ that vanish along the boundary $\partial \Omega$. Finally, $H W^{1, p}(\Omega)$ consists of those functions in $W^{1, p}(\Omega)$ that are harmonic in $\Omega$. The issue at hand is the following:

- When is the Dirichlet problem uniquely solvable on $\Omega$ for the class $W^{1, p}$ ?

In other words, given a function $f \in W^{1, p}(\Omega)$, we want to find a (uniquely determined) harmonic function $h$ in the same Sobolev space having the same boundary values on $\partial \Omega$ as does $f$. That is, $g=f-h$ should belong to $W_{0}^{1, p}(\Omega) ; h$ should be in $H W^{1, p}(\Omega)$; and in the decomposition $f=g+h$, both $g$ and $h$ are to be uniquely determined as elements of the respective subspaces of $W^{1, p}(\Omega)$. Thus our question becomes the issue of whether the following direct decomposition of the space $W^{1, p}(\Omega)$ is valid:

$$
W^{1, p}(\Omega)=W_{0}^{1, p}(\Omega)+H W^{1, p}(\Omega), \quad W_{0}^{1, p}(\Omega) \cap H W^{1, p}(\Omega)=\{0\} .
$$

A comparison with (1.2) reveals a close resemblance. The device that effects the translation between (1.2) - with $p$ and $p^{\prime}$ interchanged - and (1.4) is known as Havin's Lemma (see Section 2 below). The main result, as stated above, then implies that

- (1.4) holds for all simply connected bounded $\Omega$, provided that $p_{0}<p<p_{0}^{\prime}$.

In other words, the Dirichlet problem is uniquely solvable on bounded simply connected domains for the Sobolev class $W^{1, p}$ in the range $p_{0}<p<p_{0}^{\prime}$. 
The various versions of the main result formulated above are condensed in Theorem 4.2.

We note that Hedberg has studied the boundary values of Sobolev spaces on general domains and characterized $W_{0}^{1, p}(\Omega)$ in terms of zero boundary values, originally for $\frac{3}{2}<p<+\infty$ and, later with Wolff, generally for $1<p<+\infty$ $[24,25]$. A good source of background information on Sobolev spaces is [1].

\section{Havin's lemma, Bergman spaces, and projections}

Havin's lemma. As a matter of notation, we introduce the standard Wirtinger partial derivatives:

$$
\partial_{z}=\frac{\partial}{\partial z}=\frac{1}{2}\left(\frac{\partial}{\partial x}-i \frac{\partial}{\partial y}\right), \quad \bar{\partial}_{z}=\frac{\partial}{\partial \bar{z}}=\frac{1}{2}\left(\frac{\partial}{\partial x}+i \frac{\partial}{\partial y}\right),
$$

where $z=x+i y$.

We need the following identification of the annihilator of $A^{p^{\prime}}(\Omega)$, which constitutes a version of Havin's lemma.

Lemma 2.1. Let $1<p<+\infty$. Then the annihilator $A^{p^{\prime}}(\Omega)^{\perp}$ of $A^{p^{\prime}}(\Omega)$ in $L^{p}(\Omega)$ is the norm closure of $\partial_{z} C_{c}^{\infty}(\Omega)$ in $L^{p}(\Omega)$.

Proof. First let $f \in \partial_{z} C_{c}^{\infty}(\Omega)$, so that $f=\partial_{z} F$, where $F \in C_{c}^{\infty}(\Omega)$. For $g \in A^{p^{\prime}}(\Omega)$, we have

$$
\langle f, g\rangle_{\Omega}=\int_{\Omega} f(z) \bar{g}(z) d A(z)=\int_{\Omega} \partial_{z} F(z) \bar{g}(z) d A(z)=-\int_{\Omega} F(z) \partial_{z} \bar{g}(z) d A(z)=0,
$$

using integration by parts and the fact that $\bar{g}$ is anti-analytic on $\Omega$. It follows that $\partial_{z} C_{c}^{\infty}(\Omega)$ is contained in the annihilator $A^{p^{\prime}}(\Omega)^{\perp}$, and hence so is its norm closure in $L^{p}(\Omega)$. It remains to check that $A^{p^{\prime}}(\Omega)^{\perp}$ is no bigger than the norm closure of $\partial_{z} C_{c}^{\infty}(\Omega)$. Since $1<p<+\infty$, by duality it suffices to verify that if $g \in L^{p^{\prime}}(\Omega)$ is in the annihilator of $\partial_{z} C_{c}^{\infty}(\Omega)$, that is, if

$$
\left\langle\partial_{z} F, g\right\rangle_{\Omega}=\int_{\Omega} \partial_{z} F(z) \vec{g}(z) d A(z)=0
$$

for all $F \in C_{c}^{\infty}(\Omega)$, then $g \in A^{p^{\prime}}(\Omega)$. To this end, we again use integration by parts, and note that the above simply states that

$$
\left\langle F, \bar{\partial}_{z} g\right\rangle_{\Omega}=0, \quad F \in C_{c}^{\infty}(\Omega),
$$

in the sense of distribution theory. In other words, $\bar{\partial}_{z} g=0$ holds on $\Omega$, again as distributions. But it is well-known that this implies that $g$ is analytic, that is, $g \in A^{p^{\prime}}(\Omega)$. The proof is complete. 
Projections on Bergman spaces. We now turn to the problem of identifying a surjective projection $\Pi_{p}: L^{p}(\Omega) \rightarrow A^{p}(\Omega)$, having as kernel $A^{p^{\prime}}(\Omega)^{\perp}$, with the orthogonal projection $\Pi_{2}: L^{2}(\Omega) \rightarrow A^{2}(\Omega)$. It is clear that the operator $\Pi_{p}$ is uniquely determined by these properties. When we think of $\Pi_{p}$ as an operator $L^{p}(\Omega) \rightarrow L^{p}(\Omega)$, it has an adjoint $\Pi_{p}^{*}: L^{p^{\prime}}(\Omega) \rightarrow L^{p^{\prime}}(\Omega)$. The projection property $\Pi_{p}^{2}=\Pi_{p}$ leads to the similar property $\left(\Pi_{p}^{*}\right)^{2}=\Pi_{p}^{*}$ for the adjoint. We quickly realize that $\Pi_{p}^{*}$ has range $A^{p^{\prime}}(\Omega)$, and that its kernel equals $A^{p}(\Omega)^{\perp}$. For this reason, we sometimes write $\Pi_{p}^{*}=\Pi_{p^{\prime}}$. We restrict our attention to $1<p<2$ only, for in case $2<p<+\infty$, we consider $\Pi_{p}^{*}$ in place of $\Pi_{p}$. Let $f_{1} \in A^{p}(\Omega) \cap A^{2}(\Omega)$ and $f_{2} \in \partial_{z} C_{c}^{\infty}(\Omega)$. Then, in view of Lemma 2.1, $f_{2} \in A^{p^{\prime}}(\Omega)^{\perp} \cap A^{2}(\Omega)^{\perp}$, so that

$$
\begin{aligned}
\Pi_{p}\left(f_{1}+f_{2}\right)=\Pi_{p}\left(f_{1}\right)=f_{1}=\Pi_{2}\left(f_{1}\right) & =\Pi_{2}\left(f_{1}+f_{2}\right), \\
& f_{1} \in A^{p}(\Omega) \cap A^{2}(\Omega), f_{2} \in \partial_{z} C_{c}^{\infty}(\Omega) .
\end{aligned}
$$

Suppose now that $A^{p}(\Omega) \cap A^{2}(\Omega)$ is dense in $A^{2}(\Omega)$. Then, by Lemma 2.1,

$$
X=A^{p}(\Omega) \cap A^{2}(\Omega)+\partial_{z} C_{c}^{\infty}(\Omega)
$$

is dense in $L^{2}(\Omega)$. This means that there exists only one possible extension of $\Pi_{p} \mid X$, the restriction of $\Pi_{p}$ to $X$, which extends continuously to an operator $L^{2}(\Omega) \rightarrow L^{2}(\Omega)$ : the orthogonal projection $\Pi_{2}: L^{2}(\Omega) \rightarrow A^{2}(\Omega)$.

Let us try to develop a better statement, while changing the assumptions slightly. For each point $\lambda \in \Omega$, there exists an element $K_{\Omega, p}(\cdot, \lambda)$ of $A^{p^{\prime}}(\Omega)$ such that

$$
\Pi_{p} f(\lambda)=\left\langle f, K_{\Omega, p}(\cdot, \lambda)\right\rangle_{\Omega}=\int_{\Omega} f(z) \bar{K}_{\Omega, p}(z, \lambda) d A(z)
$$

for all functions $f \in L^{p}(\Omega)$. Suppose that $K_{\Omega, p}(\cdot, \lambda)$ belongs to $A^{2}(\Omega)$ as well, for each $\lambda \in \Omega$. (In support of this assumption, we mention that the only thing that might prevent this from being true is the possibility that $K_{\Omega, p}(\cdot, \lambda)$ drops too slowly near infinity in $\Omega$.) We deduce easily that, for $\lambda \in \Omega$,

$$
\Pi_{p} f(\lambda)=\Pi_{2} f(\lambda), \quad f \in L^{2}(\Omega) \cap L^{p}(\Omega)
$$

that is,

$$
K_{\Omega, p}(z, \lambda)=K_{\Omega}(z, \lambda), \quad(z, \lambda) \in \Omega \times \Omega,
$$

where the right hand side denotes the Bergman kernel. In other words, $\Pi_{2}$ is in fact a continuous extension of $\Pi_{p}$ to an operator $L^{2}(\Omega) \rightarrow L^{2}(\Omega)$.

An alternative, perhaps more reasonable assumption, is to suppose that the Bergman kernel $K_{\Omega}(\cdot, \lambda)$ belongs to $A^{p}(\Omega)$ as well as to $A^{2}(\Omega)$, for each $\lambda \in \Omega$. We easily deduce that, for $\lambda \in \Omega$,

$$
\Pi_{p^{\prime}} f(\lambda)=\Pi_{2} f(\lambda), \quad f \in L^{2}(\Omega) \cap L^{p^{\prime}}(\Omega),
$$


that is,

$$
K_{\Omega, p^{\prime}}(z, \lambda)=K_{\Omega}(z, \lambda), \quad(z, \lambda) \in \Omega \times \Omega,
$$

where the right hand side denotes the Bergman kernel, provided that $A^{p^{\prime}}(\Omega) \cap A^{2}(\Omega)$ is dense in $A^{p^{\prime}}(\Omega)$. In other words, $\Pi_{2}$ is the only possible continuous extension of $\Pi_{p^{\prime}}$ to an operator $L^{2}(\Omega) \rightarrow L^{2}(\Omega)$. As it is clear that

$$
K_{\Omega, p}(z, \lambda)=\bar{K}_{\Omega, p^{\prime}}(\lambda, z), \quad(z, \lambda) \in \Omega \times \Omega,
$$

the same goes for $\Pi_{p}$ as well.

We now establish that the assumption made above, that $A^{p}(\Omega) \cap A^{2}(\Omega)$ is dense in $A^{2}(\Omega)$, holds quite generally, except that we may fail to capture a single dimension.

Proposition 2.2. Let $1<p<2$. Then $A^{p}(\Omega) \cap A^{2}(\Omega)$ is either dense in $A^{2}(\Omega)$ or its closure has codimension 1 . If $\mathbb{C} \backslash \Omega$ contains an unbounded continuum, it is dense.

Proof. Consider the linear subspace

$$
\mathcal{L}=\left\{f \in A^{2}(\Omega): z f \in A^{2}(\Omega)\right\}
$$

of $A^{2}(\Omega)$. Since $1<p<2, \mathcal{L}$ is contained in $A^{p}(\Omega) \cap A^{2}(\Omega)$. We intend to show that either $\mathcal{L}$ is dense in $A^{2}(\Omega)$ or its closure has codimension one.

Consider $A^{2}(\Omega) \ominus \mathcal{L}$, the orthogonal complement of $\mathcal{L}$ in $A^{2}(\Omega)$. If it is 0 dimensional, then $\mathcal{L}$ is dense, and we are done. So, in what follows, we assume $A^{2}(\Omega) \ominus \mathcal{L}$ contains at least one nonzero vector, which we call $F$; by renormalization, we may suppose $F$ has norm 1 . This implies that the point at infinity, denoted $\infty$, cannot be a peak point for $H^{\infty}(\Omega)$; in other (more geometrical) terms, the connected component of $\mathbb{C} \cup\{\infty\} \backslash \Omega$ containing $\infty$ is the singleton $\{\infty\}$. (Recall that $\infty$ is a peak point for $H^{\infty}(\Omega)$ if there exists $q \in H^{\infty}(\Omega)$ of sup-norm 1 with

$$
q(z) \rightarrow 1 \quad \text { as } \Omega \ni z \rightarrow \infty, \quad \text { and } \quad \sup _{z \in \Omega \backslash U}|q(z)|<1
$$

for each punctured neighborhood $U$ of $\infty$.) The simple argument is (essentially) supplied in Lemma 2.1 of [3]; technical support for the argument is offered by Gamelin's and Garnett's paper [22].

We now sketch the remaining part of the proof, which is based on an idea of Browder, developed further by Gamelin and Garnett [22], and worked out in detail by Aleman in [3] in the case of a bounded domain and with $\infty$ replaced by a finite point. For positive parameters $\epsilon$ and $R$, and a function $f \in A^{2}(\Omega)$, let $\Sigma=\Sigma(\epsilon, R, F, f, \Omega)$ be the set of points $z \in \Omega$ with $|z|>R, F(z) \neq 0$, and

$$
\left|\frac{f(z)}{F(z)}-\langle f, F\rangle_{\Omega}\right|<\epsilon .
$$


We claim that

$$
\int_{\Sigma} \frac{d A(z)}{|z|^{4}}=\frac{1}{R^{2}}+o\left(\frac{1}{R^{2}}\right) \quad \text { as } R \rightarrow+\infty
$$

so that in this sense, $\Sigma$ fills almost all of the region $|z|>R$. Let us see why this implies that the closure of $\mathcal{L}$ has codimension 1 . If not, then we can find another function $G \in A^{2}(\Omega) \ominus \mathcal{L}$, which has norm 1 and is orthogonal to $F$. Taking a look at (2.3) with $f=G$, and then reversing the roles of $F$ and $G$, we find that

$$
\left|\frac{G(z)}{F(z)}\right|<\epsilon, \quad\left|\frac{F(z)}{G(z)}\right|<\epsilon
$$

must hold simultaneously for many points in $\Omega$ of large modulus, which is clearly impossible for $0<\epsilon<1$.

The verification of (2.4) is carried out as in [3], with necessary modifications which arise because the point is $\infty$ and the complement $\mathbb{C} \backslash \Omega$ may have zero area.

It seems likely that an analogous statement is valid regarding the closure of $A^{p^{\prime}}(\Omega) \cap A^{2}(\Omega)$ in $A^{p^{\prime}}(\Omega)$, for $1<p<2$.

Let us see where Proposition 2.2 leaves us as for the duality problem. Let $F \in A^{2}(\Omega)$ be the function appearing in the proof of Proposition 2.2 and let $1<p<2$. If $F$ is in $A^{p}(\Omega)$, then $A^{2}(\Omega) \cap A^{p}(\Omega)$ is dense in $A^{2}(\Omega)$, and we are done. There is reason to believe that $A^{2}(\Omega) \cap A^{p}(\Omega)$ is always dense in $A^{2}(\Omega)$. After all, if $\infty$ is an interior point in the complement $\mathbb{C} \backslash \Omega$, then $A^{2}(\Omega) \cap A^{\mathcal{P}}(\Omega)=A^{2}(\Omega)$; and the same holds if $\infty$ is a boundary point of $\Omega$ in the extended plane, provided the boundary $\partial \Omega$ is sufficiently "massive" near $\infty$ (for instance, if it contains a continuum with one end point $\infty$ ). On the other hand, if $\partial \Omega$ is "thin" near $\infty$, we consider the extreme case that $\infty$ is an interior point, and note that then we must have

$$
F(z)=O\left(\frac{1}{|z|^{2}}\right), \quad|z| \rightarrow+\infty,
$$

in order for $F$ to belong to $A^{2}(\Omega)$. So, we get $F \in A^{p}(\Omega)$ automatically.

We collect our observations in the following.

Proposition 2.3. Let $1<p<2$ and let $\Omega$ be a domain in $\mathbb{C}$ such that $A^{p}(\Omega) \cap A^{2}(\Omega)$ is dense in $A^{2}(\Omega)$. Suppose there exists a projection $\Pi_{p}: L^{p}(\Omega) \rightarrow$ $A^{p}(\Omega)$ which is the identity on $A^{p}(\Omega)$ and whose kernel is $A^{p^{\prime}}(\Omega)^{\perp}$. Suppose, moreover, that for each $\zeta \in \Omega$, the function $K_{\Omega, p}(\cdot, \zeta)$ defined by (2.2) belongs to $A^{2}(\Omega)$. Then $\Pi_{p}$ is the Bergman projection $\Pi_{2}: L^{2}(\Omega) \rightarrow A^{2}(\Omega)$, in the sense that 
$\Pi_{p} f=\Pi_{2} f$ for all $f \in L^{2}(\Omega) \cap L^{p}(\Omega)$. Furthermore, in terms of the Bergman kernel $K_{\Omega}(z, \zeta)$,

$$
\Pi_{p} f(\zeta)=\int_{\Omega} f(z) \bar{K}_{\Omega}(z, \zeta) d A(z), \quad \zeta \in \Omega
$$

for all $f \in L^{p}(\Omega)$. Finally, for each fixed $\zeta \in \Omega$, the function $K_{\Omega}(\cdot, \zeta)$ belongs to $A^{p}(\Omega) \cap A^{p^{\prime}}(\Omega)$.

For $2<p<+\infty$, an analogous set of assertions holds.

The statement that $K_{\Omega, p}(\cdot, \zeta)=K_{\Omega}(\cdot, \zeta)$ belongs to $A^{p}(\Omega)$ is deduced from the self-adjointness of $\Pi_{2}$ and the known properties of the operator $\Pi_{p}^{*}=\Pi_{p^{\prime}}$.

We should also consider the converse situation, when we know the Bergman projection extends continuously to $L^{p}(\Omega)$, and would like to know that it is a projection $L^{p}(\Omega) \rightarrow A^{p}(\Omega)$ whose kernel equals $A^{p^{\prime}}(\Omega)^{\perp}$.

Proposition 2.4. Let $1<p<+\infty$ and let $\Omega$ be a domain in $\mathrm{C}$ such that $A^{p}(\Omega) \cap A^{2}(\Omega)$ is dense in $A^{p}(\Omega)$, and $A^{p^{\prime}}(\Omega) \cap A^{2}(\Omega)$ is dense in $A^{p^{\prime}}(\Omega)$. Let $\Pi_{2}: L^{2}(\Omega) \rightarrow A^{2}(\Omega)$ be the orthogonal projection, given by the integral formula

$$
\Pi_{2} f(\zeta)=\left\langle f, K_{\Omega}(\cdot, \zeta)\right\rangle_{\Omega}=\int_{\Omega} f(z) \bar{K}_{\Omega}(z, \zeta) d A(z), \quad \zeta \in \Omega .
$$

Suppose that for each $\zeta \in \Omega, K_{\Omega}(\cdot, \zeta)$ belongs to $A^{p}(\Omega) \cap A^{p^{\prime}}(\Omega)$, so that $\Pi_{2} f(\zeta)$ makes sense for $f \in L^{p}(\Omega)$ and for $f \in L^{p^{\prime}}(\Omega)$. Suppose, moreover, that $\Pi_{2}$ extends continuously to an operator $\Pi_{p}: L^{p}(\Omega) \rightarrow A^{p}(\Omega)$. Then $\Pi_{p}$ is the identity on $A^{p}(\Omega)$, and its kernel is $A^{p^{\prime}}(\Omega)^{\perp}$.

Proof. We are to check that $\Pi_{p} f=f$ for $f \in A^{p}(\Omega)$, and that $\Pi_{p} g=0$ if and only if $g \in A^{p^{\prime}}(\Omega)^{\perp}$. By duality, the latter statement follows once we know that $\Pi_{p}^{*} h=h$ holds for all $h \in A^{p^{\prime}}(\Omega)$, where $\Pi_{p}^{*}$ is the adjoint to $\Pi_{p}$, thought of as acting $\Pi_{p}: L^{p}(\Omega) \rightarrow L^{p}(\Omega)$. We realize that $\Pi_{p}^{*}$ supplies a (unique) continuous extension of $\Pi_{2}$ to an operator $L^{p^{\prime}}(\Omega) \rightarrow A^{p^{\prime}}(\Omega)$, which we can call $\Pi_{p^{\prime}}$. In view of the density assumptions made, these assertions are immediate consequences of the continuity of $\Pi_{p}$ and $\Pi_{p^{\prime}}$.

\section{Reduction to weighted spaces on the disk}

The Bergman kernel and conformal mapping. Let $\Omega$ be a simply connected subdomain of $\mathbb{C}$ which is not the whole plane. By the Riemann mapping theorem, there exists a conformal mapping $\phi: \Omega \rightarrow \mathbb{D}$ of $\Omega$ onto the open unit 
disk $\mathbb{D}$. It is well-known [7] that the Bergman kernel for $\Omega$ can be expressed conveniently in terms of $\phi$ :

$$
K_{\Omega}(z, \zeta)=\frac{\phi^{\prime}(z) \bar{\phi}^{\prime}(\zeta)}{(1-\phi(z) \bar{\phi}(\zeta))^{2}}, \quad(z, \zeta) \in \Omega \times \Omega .
$$

In this representation, it is not important which Riemann map $\phi$ we use. We see immediately the connection between the Bergman kernel for $\Omega$ and that of the unit disk $\mathbb{D}$ :

$$
K_{\mathbb{D}}(z, \zeta)=\frac{1}{(1-z \bar{\zeta})^{2}}, \quad(z, \zeta) \in \mathbb{D} \times \mathbb{D} \text {. }
$$

The issue at hand is whether

$$
\Pi_{\Omega} f(z)=\int_{\Omega} K_{\Omega}(z, \zeta) f(\zeta) d A(\zeta), \quad z \in \Omega,
$$

defines a continuous projection $L^{p}(\Omega) \rightarrow A^{p}(\Omega)$. Let us use $\varphi=\phi^{-1}: \mathbb{D} \rightarrow \Omega$ to transform the problem into one for the unit disk. We introduce the operator

$$
\Pi_{*} f(z)=\varphi^{\prime}(z) \Pi_{\Omega}\left(\phi^{\prime} f \circ \phi\right) \circ \varphi(z), \quad z \in \mathbb{D},
$$

for functions $f$ on the unit disk. A calculation involving a Moebius shift of coordinates then reveals that

$$
\begin{aligned}
& \Pi_{*} f(z)=\varphi^{\prime}(z) \Pi_{\Omega}\left(\phi^{\prime} f \circ \phi\right) \circ \varphi(z)=\varphi^{\prime}(z) \int_{\Omega} K_{\Omega}(\varphi(z), \zeta) f \circ \phi(\zeta) \phi^{\prime}(\zeta) d A(\zeta) \\
= & \int_{\Omega} \frac{1}{(1-z \bar{\phi}(\zeta))^{2}} f \circ \phi(\zeta)\left|\phi^{\prime}(\zeta)\right|^{2} d A(\zeta)=\int_{\mathbb{D}} \frac{1}{(1-z \bar{\zeta})^{2}} f(\zeta) d A(\zeta), \quad z \in \mathbb{D},
\end{aligned}
$$

so that $\Pi_{*}=\Pi_{\mathbb{D}}$, the Bergman projection on the unit disk. Scrutinizing these formulas, we realize that $\Pi_{\Omega}$ defines a continuous projection $L^{p}(\Omega) \rightarrow A^{p}(\Omega)$ if and only if $\Pi_{\mathbb{D}}$ defines a continuous projection $L^{p}\left(\mathbb{D}, \omega^{p}\right) \rightarrow A^{p}\left(\mathbb{D}, \omega^{p}\right)$, where we are dealing with weighted spaces; the weight is $\omega=\omega_{p}=\left|\varphi^{\prime}\right|^{2 / p-1}$, the space $L^{p}\left(\mathbb{D}, \omega^{p}\right)$ consists of (equivalence classes of) Lebesgue measurable functions $f$ with

$$
\left.\|f\|_{L^{p}\left(\mathbb{D}, \omega^{p}\right)}=\left(\int_{\mathbb{D}}|f(z)|^{p} \omega_{i} z\right)^{p} d A(z)\right)^{1 / p}<+\infty
$$

and $A^{p}\left(\mathbb{D}, \omega^{p}\right)$ is the closed subspace of $L^{p}\left(\mathbb{D}, \omega^{p}\right)$ consisting of those functions that are holomorphic on $\mathbb{D}$. In case $\omega \in L^{p}(\mathbb{D})$, the Békollé-Bonami theorem [5] describes the weights $\omega$ for which this happens in terms of a Muckenhoupt $\left(A_{p}\right)$ condition. From the text and the references of [38], it appears that Solovyov had the same result at approximately the same time as Békollé and Bonami. By the Koebe-Bieberbach distortion theorem $[11,20,36]$,

$$
\left|\varphi^{\prime}(0)\right| \frac{1-|z|}{(1+|z|)^{3}} \leq\left|\varphi^{\prime}(z)\right| \leq\left|\varphi^{\prime}(0)\right| \frac{1+|z|}{(1-|z|)^{3}}, \quad z \in \mathbb{D},
$$


we are guaranteed that $\omega=\omega_{p} \in L^{p}(\mathbb{D})$ in the interval $\frac{5}{3}<p<3$. However, more sophisticated results yield a larger interval. Indeed, the estimate

$$
\int_{-\pi}^{\pi}\left|\varphi^{\prime}\left(r e^{i \theta}\right)\right|^{q} d \theta=O\left(\frac{1}{(1-r)^{3 q-1+\epsilon}}\right) \quad \text { as } r \rightarrow 1
$$

which is due to Feng and MacGregor $[21,36]$ and valid for positive (small) $\epsilon$ and $\frac{2}{5} \leq q<+\infty$, leads to the conclusion that $\omega \in L^{p}(\mathbb{D})$ for $p$ in the interval $\frac{4}{3}<p \leq 2$. Using the estimate of Theorem 8.5 [36], we may also extend the interval to the right, to incorporate all $p$ with $2 \leq p<(7+\sqrt{7}) / 3 \approx 3.21$. Thus $\omega \in L^{p}(\mathbb{D})$ for $\frac{4}{3}<p<(7+\sqrt{7}) / 3$. The celebrated Brennan conjecture asserts that this holds on the interval $\frac{4}{3}<p<4$. The best right hand side bound currently known is $\approx 3.422$, which was obtained by Bertilsson [8, 9]. For our purposes, however, it suffices to deal with the interval $\frac{4}{3}<p \leq 2$, as the boundedness of $\Pi_{\Omega}$ as an operator $L^{p}(\Omega) \rightarrow A^{p}(\Omega)$ implies the boundedness of the adjoint, which is the same operator, only this time mapping boundedly $L^{p^{\prime}}(\Omega) \rightarrow A^{p^{\prime}}(\Omega)$. Note that 4 is the exponent dual to $\frac{4}{3}$.

Békollé-Bonami's theorem of Muckenhoupt type for the disk. Now let $\frac{4}{3}<p \leq 2$. By the above, $\omega=\omega_{p} \in L^{p}(\mathbb{D})$. Set

$$
\langle f\rangle_{E}=\frac{1}{|E|_{A}} \int_{E} f(z) d A(z)
$$

for measurable subsets $E$ of positive area and area-summable functions $f$ on $E$, where $|E|_{A}$ stands for the normalized area of $E$. The (area) Muckenhoupt $\left(A_{p}\right)$ condition for the weight $\omega^{p}$ reads

$$
\sup _{Q}\left\langle\omega^{p}\right\rangle_{Q}^{1 / p}\left\langle\omega^{-p^{\prime}}\right\rangle_{Q}^{1 / p^{\prime}}<+\infty
$$

with the supremum ranging over all Carleson "squares" $Q$ in $\mathbb{D}$. The BékolléBonami theorem then says that $\Pi_{\Omega}$ defines a continuous projection $L^{p}(\Omega) \rightarrow A^{p}(\Omega)$ if and only if

$$
\sup _{Q}\left\langle\left|\varphi^{\prime}\right|^{2-p}\right\rangle_{Q}\left\langle\left|\varphi^{\prime}\right|^{(p-2) /(p-1)}\right\rangle_{Q}^{p-1}<+\infty .
$$

By the Feng-MacGregor theorem [21, 36],

$$
\int_{\mathbb{D}}\left|\varphi^{\prime}(z)\right|^{2-p} d A(z) \leq C(p)\left|\varphi^{\prime}(0)\right|^{2-p}, \quad \frac{4}{3}<p \leq 2,
$$

for some positive constant $C(p)$ that only depends on $p$. Suppose, moreover, that

$$
\int_{\mathrm{D}}\left|\varphi^{\prime}(z)\right|^{-q} d A(z) \leq C^{\prime}(q)\left|\varphi^{\prime}(0)\right|^{-q}, \quad 0 \leq q<q_{1},
$$


holds for some positive number $q_{1}$, where the constant $C^{\prime}(q)$ is positive and depends only on $q$. All efforts at proving Brennan's conjecture so far have resulted in estimates of the type (3.3), which may look (superficially) stronger. So, for instance, by Bertilsson [9], we have (3.3) for $q_{1} \approx 1.422$. Combining the estimates (3.2) and (3.3), we arrive at

$$
\begin{aligned}
& \int_{\mathbb{D}}\left|\varphi^{\prime}(z)\right|^{2-p} d A(z)\left(\int_{\mathbb{D}}\left|\varphi^{\prime}(z)\right|^{(p-2) /(p-1)} d A(z)\right)^{p-1} \\
& \leq C(p) C^{\prime}\left(\frac{2-p}{p-1}\right)^{p-1}, \quad p_{1}<p \leq 2,
\end{aligned}
$$

where $\left(2-p_{1}\right) /\left(p_{1}-1\right)=q_{1}$; numerically, $p_{1} \approx 1.413$.

We now use the Moebius invariance of the class of conformal mappings $\varphi$ considered. For a Moebius automorphism $\psi$ of the unit disk $\mathbb{D}, \varphi \circ \psi$ is just another conformal mapping to which the above estimate (3.4) applies. This implies that we also have

$$
\begin{gathered}
\int_{\mathbb{D}}\left|\varphi^{\prime} \circ \psi(z) \psi^{\prime}(z)\right|^{2-p} d A(z)\left(\int_{\mathbb{D}}\left|\varphi^{\prime} \circ \psi(z) \psi^{\prime}(z)\right|^{(p-2) /(p-1)} d A(z)\right)^{p-1} \\
\leq C(p) C^{\prime}\left(\frac{2-p}{p-1}\right)^{p-1}, \quad p_{1}<p \leq 2 .
\end{gathered}
$$

Assuming the automorphism to be involutive, that is, $\psi \circ \psi(z)=z$, we have after a shift of coordinates

$$
\begin{gathered}
\int_{\mathbb{D}}\left|\varphi^{\prime}(z)\right|^{2-p}\left|\psi^{\prime}(z)\right|^{p} d A(z)\left(\int_{\mathbb{D}}\left|\varphi^{\prime}(z)\right|^{(p-2) /(p-1)}\left|\psi^{\prime}(z)\right|^{p /(p-1)} d A(z)\right)^{p-1} \\
\leq C(p) C^{\prime}\left(\frac{2-p}{p-1}\right)^{p-1}, \quad p_{1}<p \leq 2 .
\end{gathered}
$$

Involutive automorphisms $\psi$ have the form

$$
\psi(z)=\frac{\lambda-z}{1-\bar{\lambda} z}, \quad z \in \mathbb{D}
$$

with $\lambda \in \mathbb{D}$, so that

$$
\psi^{\prime}(z)=-\frac{1-|\lambda|^{2}}{(1-\bar{\lambda} z)^{2}}, \quad z \in \mathbb{D}
$$

and

$$
\left|\psi^{\prime}(z)\right|^{2}=\frac{\left(1-|\lambda|^{2}\right)^{2}}{11-\left.\bar{\lambda} z\right|^{4}}, \quad z \in \mathbb{D}
$$

According to [26, pp. 7-8], we have the asymptotic behavior

$$
\int_{\mathbb{D}}\left|\psi^{\prime}(z)\right|^{r} d A(z) \asymp \frac{1}{\left(1-|\lambda|^{2}\right)^{r-2}}
$$


as $|\lambda| \rightarrow 1$, provided that $1<r<+\infty$. In view of this, we can write (3.6) in the form

$$
\left\langle\left|\varphi^{\prime}\right|^{2-p}\right\rangle_{\left|\psi^{\prime}\right|^{p}}\left(\left\langle\left|\varphi^{\prime}\right|^{(p-2) /(p-1)}\right\rangle_{\left|\psi^{\prime}\right|^{p /(p-1)}}\right)^{p-1} \leq C^{\prime \prime}(p), \quad p_{1}<p \leq 2,
$$

for some positive constant $C^{\prime \prime}(p)$ which depends only on $p$. Here, for an areasummable weight $\omega$, we write

$$
\langle f\rangle_{\omega}=\int_{\mathbb{D}} f(z) \omega(z) d A(z) / \int_{\mathbb{D}} \omega(z) d A(z)
$$

for the weighted average of the function $f$ on $\mathbb{D}$. If we scrutinize the behavior of $\left|\psi^{\prime}\right|$ on Carleson "squares" $Q$ in $\mathbb{D}$, it becomes clear that (3.7) implies the area Muckenhoupt $\left(A_{p}\right)$ condition (3.1).

Theorem 3.1. Let $\Omega$ be a simply connected domain in the complex plane, $\Omega \neq$ C. Then for $p$ with $p_{1}<p<p_{1}^{\prime}$, where $p_{1} \approx 1.413$ and $p_{1}^{\prime} \approx 3.422, A^{p}(\Omega)^{*}=A^{p^{\prime}}(\Omega)$, and the Bergman projection defines a bounded projection $L^{p}(\Omega) \rightarrow A^{p}(\Omega)$, which is the identity on $A^{p}(\Omega)$ and has kernel $A^{p^{\prime}}(\Omega)^{\perp}$.

Proof. It is clear from the above that the Bergman projection defines a bounded operator $\Pi_{p}: L^{p}(\Omega) \rightarrow A^{p}(\Omega)$ in the indicated range of $p$. It remains to show that $\Pi_{p}$ is the identity on $A^{p}(\Omega)$ and to identify the kernel. By Proposition 2.4, it suffices to check that $A^{2}(\Omega) \cap A^{p}(\Omega)$ is dense in $A^{p}(\Omega)$ and that $A^{2}(\Omega) \cap A^{p^{\prime}}(\Omega)$ is dense in $A^{p^{\prime}}(\Omega)$. After making a conformal change of coordinates involving the function $\varphi: \mathbb{D} \rightarrow \Omega$, and performing a division by $\varphi^{\prime}$, the issue is whether $A^{p}\left(\mathbb{D},\left|\varphi^{\prime}\right|^{2-p}\right) \cap A^{2}(\mathbb{D})$ is dense in $A^{p}\left(\mathbb{D},\left|\varphi^{\prime}\right|^{2-p}\right)$, and whether the same is true with $p$ replaced by $p^{\prime}$. As the indicated interval of $p$ 's is dually invariant, it suffices to deal with $p$ alone. The function $\left|\varphi^{\prime}\right|^{2-p}$ is in $L^{1}(\mathbb{D})$, by (3.2) and (3.3); hence, it suffices to show that the polynomials are dense in $A^{p}\left(\mathbb{D},\left|\varphi^{\prime}\right|^{2-p}\right)$. This is equivalent to showing that the function $\left(\varphi^{\prime}\right)^{2 / p-1}$ is cyclic in $A^{p}(\mathbb{D})$, i.e., that its polynomial multiples are dense in $A^{p}(\mathbb{D})$. As a matter of fact, (3.2) and (3.3) show that $\left(\varphi^{\prime}\right)^{2 / p-1}$ is in a slightly better Bergman space $A^{r}(\mathbb{D})$ for some $r, p<r<+\infty$. In view of the estimate from below of the modulus of $\left(\varphi^{\prime}\right)^{2 / p-1}$ afforded by the Koebe-Bieberbach theorem, say, and of the theorem of Shapiro on cyclicity, later refined by Brown and Korenblum (Theorem 7.3 in [26]; also compare with the counterexample in [12] to the conjecture that the slightly-better space requirement could be dropped), it follows that $\left(\varphi^{\prime}\right)^{2 / p-1}$ is cyclic in $A^{p}(\mathbb{D})$. The proof is complete.

We also have the following conclusion from our study.

Theorem 3.2. The following three conditions are equivalent. 
(a) Brennan's conjecture holds, that is, $1 / \varphi^{\prime}$ belongs to $A^{p}(\mathbb{D})$ for all $p$ with $0<p<2$ and for all univalent functions $\varphi$ on $\mathbb{D}$,

(b) The Bergman projection $\Pi_{\Omega}: L^{2}(\Omega) \rightarrow A^{2}(\Omega)$ extends to a continuous projection $L^{p}(\Omega) \rightarrow A^{p}(\Omega)$ for all $p$ with $\frac{4}{3}<p<4$ and for all simply connected $\Omega$ other than $\mathbb{C}$ itself.

(c) With respect to the dual action induced by $A^{2}(\Omega)$, the dual space of $A^{p}(\Omega)$ is $A^{p^{\prime}}(\Omega)$ for all $p$ with $\frac{4}{3}<p<4$ and for all simply connected $\Omega$ other than $\mathbb{C}$ itself.

Proof. We first consider the implication $(a) \Rightarrow(b)$. By the above argument involving the Békollé-Bonami theorem, it suffices to establish (3.3) with $q_{1}=2$. Bertilsson [9] has shown that Brennan's conjecture (a) actually implies the stronger assertion that

$$
\int_{-\pi}^{\pi} \frac{d \theta}{\left|\varphi^{\prime}\left(r e^{i \theta}\right)\right|^{2}} \leq \frac{K}{\left|\varphi^{\prime}(0)\right|^{2}} \frac{1}{1-r}
$$

for some absolute constant $K$. In view of this, the estimate (3.3) easily follows.

The implication $(b) \Rightarrow(c)$ is a consequence of the discussion in the introduction, as well as that of Section 2.

We turn finally to the implication (c) $\Rightarrow(a)$. We restrict our attention to bounded simply connected $\Omega$. By the argument presented in the introduction, as well as in Section 2, condition (c) is equivalent to the boundedness of the Bergman projection $\Pi_{\Omega}: L^{p}(\Omega) \rightarrow A^{p}(\Omega)$ for all $p$ with $\frac{4}{3}<p \leq 4$, which in its turn is equivalent to having (3.1) for all $p$ with $\frac{4}{3}<p \leq 2$. But then the function $\left|\varphi^{\prime}\right|^{-q}$ is at least area-summable on all "Carleson squares", and consequently on all of $\mathbb{D}$, for $q=(2-p) /(p-1)$, which is free in the range $0 \leq q<2$. From this, (a) is immediate for bounded univalent $\varphi$. However, according to Makarov [32], Brennan's conjecture follows for arbitrary univalent functions once it is known for bounded univalent functions. We are done.

A class of Volterra-type operators. We want to describe a method that allows us to use information of the type that if $F$ belongs to the Bloch space $\mathcal{B}(\mathbb{D})$, then $e^{\mu F}$ is in $A^{2}(\mathbb{D})$ for small complex constants $\mu$; it constitutes an area version of the John-Nirenberg theorem. This is relevant to us because we want certain negative powers of $\varphi^{\prime}$ to be in $A^{2}(\mathbb{D})$, while it is possible to obtain reasonably adequate information regarding the Bloch space function $\log \varphi^{\prime}$; here, $\varphi: \mathbb{D} \rightarrow \mathbb{C}$ is a univalent function. The hope is that it may be possible to obtain more detailed information that can lead to better estimates than those known today.

Given an analytic function $F$ in the unit disk $\mathbb{D}$, we consider the Volterra-type operator

$$
T_{F} f(z)=\int_{0}^{z} F^{\prime}(\zeta) f(\zeta) d \zeta, \quad z \in \mathbb{D}
$$


where $f \in A^{2}(\mathbb{D})$. This family of operators was originally considered by Pommerenke [35]. As only the derivative of $F$ appears in the definition of the operator, we may consider only $F$ with $F(0)=0$. It is well-known [4] that $T_{F}$ is a bounded operator on $A^{2}(\mathbb{D})$ if and only if $F$ belongs to the Bloch space $\mathcal{B}(\mathbb{D})$. For a Bloch function $F$, let $\sigma\left(T_{F}\right)$ denote the spectrum of the operator $T_{F}: A^{2}(\mathbb{D}) \rightarrow A^{2}(\mathbb{D})$. The reason why $T_{F}$ is relevant to the situation at hand is that if a point $\lambda$ is in the unbounded component of $\mathbb{C} \backslash \sigma\left(T_{F}\right)$, then the function $\exp (F / \lambda)$ belongs to $A^{2}(\mathbb{D})$. The argument for this is quite simple. For large $|\lambda|$, expand the resolvent in a Neumann series

$$
\left(I-\lambda^{-1} T_{F}\right)^{-1}=I+\lambda^{-1} T_{F}+\lambda^{-2} T_{F}^{2}+\cdots,
$$

where $I$ stands for the identity operator, and apply it to the constant function 1; note that

$$
T_{F}^{n} 1=\frac{1}{n !} F^{n}, \quad n=0,1,2,3, \ldots,
$$

if we make the normalization $F(0)=0$ as above. It follows that

$$
\left(I-\lambda^{-1} T_{F}\right)^{-1} 1=1+\lambda^{-1} F+\frac{1}{2 !} \lambda^{-2} F^{2}+\frac{1}{3 !} \lambda^{-3} F^{3}+\cdots=\exp (F / \lambda),
$$

so the assertion follows for large $|\lambda|$. For general $\lambda$, the result follows by analytic continuation.

If we are to use the above trick to solve Brennan's conjecture and, by Theorem 3.2 , to solve the duality problem as well as the problem of the boundedness of the Bergman projection, then, by (3.1), we need to know that for each $p$ in the interval $\frac{4}{3}<p<2$, the points $1 /(1-p / 2)$ and $(p-1) /(p / 2-1)$ are in the unbounded component of the resolvent set $\mathbb{C} \backslash \sigma\left(T_{F}\right)$, with

$$
F(z)=\log \frac{\varphi^{\prime}(z)}{\varphi^{\prime}(0)}, \quad z \in \mathbb{D} .
$$

In other words, the intervals $]-\infty,-1[$ and $[3,+\infty[$ should form part of the resolvent set. Looking at the Koebe function as a special case, we find that this is so in that very special instance; more importantly, we also see that no bigger interval would work. This suggests that the answer to the following problem might be affirmative.

Problem 3.3. For the above $F$, with $\varphi$ univalent in $\mathbb{D}$, is the spectrum $\sigma\left(T_{F}\right)$ contained in the disk

$$
\overline{\mathbb{D}}(1,2)=\{z \in \mathbb{C}:|z-1| \leq 2\} ?
$$

In other words (by the spectral radius formula), does the estimate $\left\|\left(I-T_{F}\right)^{n}\right\| \leq$ $(2+\epsilon)^{n}$ hold for large $n$ with fixed (small) positive $\epsilon$ ? 
It is well-known that for bounded univalent $\varphi$, better growth estimates of $\varphi^{\prime}$ are possible than for general univalent functions. In particular, $\varphi^{\prime} \in A^{2}(\mathbb{D})$ then, which suggests the next problem.

Problem 3.4. For the above $F$, with $\varphi$ bounded and univalent in $\mathbb{D}$, is the spectrum $\sigma\left(T_{F}\right)$ contained in the closed unit disk $\mathbb{D}$ ? In other words, does the estimate $\left\|T_{F}^{n}\right\| \leq(1+\epsilon)^{n}$ hold for large $n$ with fixed (small) positive $\epsilon$ ?

Let us take a look at what these problems ask for in addition to the desired conclusion that (3.1) hold on the required interval $\frac{4}{3}<p<4$. That $\lambda$ belongs to the resolvent set $\mathbb{C} \backslash\left(T_{F}\right)$ means that for each $f \in A^{2}(\mathbb{D})$, the equation

$$
\left(I-\lambda^{-1} T_{F}\right) g=f
$$

has a unique solution $g \in A^{2}(\mathbb{D})$. Written out explicitly, this equation reads

$$
g(z)-\lambda^{-1} \int_{0}^{z} F^{\prime}(\zeta) g(\zeta) d \zeta=f(z), \quad z \in \mathbb{D},
$$

which we easily solve:

$$
g(z)=f(0) e^{F(z) / \lambda}+e^{F(z) / \lambda} \int_{0}^{z} f^{\prime}(\zeta) e^{-F(\zeta) / \lambda} d \zeta, \quad z \in \mathbb{D} .
$$

So, we need the following two things:

$$
e^{F(z) / \lambda} \in A^{2}(\mathbb{D}), \quad \partial_{z}\left(e^{-F / \lambda} A^{2}(\mathbb{D})\right)=e^{-F / \lambda} \partial_{z} A^{2}(\mathbb{D}),
$$

where $\partial_{z}$ stands for differentiation with respect to $z$, as usual. The second condition asks that the operators of differentiation and multiplication by $e^{-F / \lambda}$ "commute" in the weak sense that the two products have equal ranges. It is not clear how much this second condition adds to the first; however, for the very simple Bloch function $F(z)=\log (1-z)$, an explicit computation shows that the second condition in (3.8) is fulfilled automatically when the first is.

In any case, (3.8) suggests the following very general problem.

Problem 3.5. Let $\mathcal{H}=\mathcal{H}(X)$ be a Hilbert space of functions on the set $X$. Consider two operators $P$ and $Q$; if they are both bounded, we say that they rangecommute if $P Q(\mathcal{H})=Q P(\mathcal{H})$. If they are unbounded, we require them to be densely defined, in such a way that $P Q$ and $Q P$ are densely defined, too. We also need both $P Q$ and $Q P$ to extend continuously to $\mathcal{H}$ in such a way that the range consists of functions on $X$. This allows us to speak of range-commuting operators in a much more general situation. The problem is to characterize the range-commuting pairs of operators on $\mathcal{H}$. 
(a) A classical concrete situation is when $\mathcal{H}=L^{2}(\mathbb{T}), X=\mathbb{T}, P$ is the orthogonal projection $L^{2}(\mathbb{T}) \rightarrow H^{2}(\mathbb{D})$, and $Q$ is the operator of multiplication by an outer function q. The Helson-Szegö theorem [27, 23] answers this problem in terms of a decomposition of $\log |q|$, and the Hunt-Muckenhoupt-Wheeden theorem $[28,23]$ answers it in terms of the $\left(A_{2}\right)$ condition for $|q|$.

(b) The above-mentioned situation corresponds to $\mathcal{H}=A^{2}(\mathbb{D}), X=\mathbb{D}, P=\partial_{z}$, and $Q$ is again multiplication by a zero-free analytic function on $\mathbb{D}$. The problem does not change much if we replace $P=\partial_{z}$ with the multiplicative differentiation operator $P=z \partial_{z}$. Now, the property that $z \partial_{z}$ shares with the Cauchy projection mentioned in part (a) is the rotation invariance; in other words, both operators are (Taylor) coefficient multipliers.

\section{The Dirichlet problem on bounded domains}

Applications to the Dirichlet problem. Let $\Omega$ be a bounded domain in the complex plane and let $1<p<+\infty$. The Sobolev spaces $W^{1, p}(\mathbb{C}), W^{1, p}(\Omega)$, $W_{0}^{1, p}(\Omega)$, and $H W^{1, p}(\Omega)$ were all defined in the introduction. We need the following elementary lemma.

Lemma 4.1. Suppose $f_{1}, f_{2}, f_{3}, \ldots$ is a sequence of functions in $C_{c}^{\infty}(\Omega)$ which converges in the norm of $W^{1, p}(\mathbb{C})$ to a function $f_{\infty} \in W^{\mathbf{1}, p}(\mathbb{C})$. If $\left.f_{\infty}\right|_{\Omega}=0$ area-almost everywhere, then $f_{\infty}=0$ as an element of $W^{1, p}(\mathbb{C})$.

Proof. By a standard Sobolev space argument, we may pick a subsequence of the given sequence that converges area-almost everywhere to the limit $f_{\infty}$. It follows that $f_{\infty}(z)=0$ area-almost everywhere in $\mathbb{C}$, whence the assertion is immediate.

As we mentioned in the introduction, this allows us to view $W_{0}^{1, p}(\Omega)$ as a (closed) subspace of $W^{1, p}(\Omega)$. We now come to the main theorem in this section.

Theorem 4.2. Let $\Omega$ be a bounded domain in $\mathbb{C}$, and let $1<p<+\infty$. The following three conditions are equivalent.

(a) With respect to the duality of $A^{2}(\Omega), A^{p^{\prime}}(\Omega)^{*}=A^{p}(\Omega)$.

(b) $L^{p}(\Omega)=A^{p}(\Omega)+A^{p^{\prime}}(\Omega)^{\perp}$, with $A^{p}(\Omega) \cap A^{p^{\prime}}(\Omega)^{\perp}=\{0\}$.

(c) The Bergman projection $\Pi_{\Omega}: L^{2}(\Omega) \rightarrow A^{2}(\Omega)$ defines a bounded projection $L^{p}(\Omega) \rightarrow A^{p}(\Omega)$.

(d) $W^{1, p}(\Omega)=H W^{1, p}(\Omega)+W_{0}^{1, p}(\Omega)$, with $H W^{1, p}(\Omega) \cap W_{0}^{1, p}(\Omega)=\{0\}$. 
(e) The Dirichlet problem is uniquely solvable on $\Omega$ with respect to the Sobolev class $W^{1, p}$.

Proof. Condition (e) is to be interpreted in the sense of the introduction, where we declared that it was to mean precisely (d).

The equivalences (a) $\Leftrightarrow(b) \Leftrightarrow(c)$ were (more or less) obtained in the introduction. Hence it remains to establish the equivalence $(b) \Leftrightarrow(d)$.

We first consider the implication (d) $\Rightarrow(\mathrm{b})$. Let $f \in L^{p}(\Omega)$, and extend this function to a function $\tilde{f}$, which is compactly supported in $\mathbb{C}$ and belongs to the space $L^{p}(\mathbb{C})$. Consider the function

$$
g(z)=\overline{\mathfrak{C}}[\tilde{f}](z)=\int_{\mathbb{C}} \frac{\tilde{f}(\zeta)}{\bar{z}-\bar{\zeta}} d A(\zeta), \quad z \in \mathbb{C},
$$

assuming that the extension $\tilde{f}$ is chosen so that $g(z)=O\left(|z|^{-2}\right)$ as $|z| \rightarrow+\infty$; what is needed is that $\tilde{f}$ should have integral 0 over $\mathbb{C}$. Then $g \in L^{p}(\mathbb{C})$, and $\partial_{z} g=\tilde{f}$ holds in $\mathbb{C}$ in the sense of distribution theory. Moreover, from the boundedness of the Beurling transform on $L^{p}(\mathbb{C})[2,16]$ (see also $[39,40]$ ), we infer that $\bar{\partial}_{z} g \in L^{p}(\mathbb{C})$. The function $g$ is then an element of the Sobolev space $W^{1, p}(\mathbb{C})$, so that its restriction $\left.g\right|_{\Omega}$ belongs to $W^{1, \nu}(\Omega)$. By $(\mathrm{d}),\left.g\right|_{\Omega}$ has a unique decomposition $\left.g\right|_{\Omega}=g_{1}+g_{2}$, where $g_{1} \in H W^{1, p}(\Omega)$ and $g_{2} \in W_{0}^{1, p}(\Omega)$. Applying the operator $\partial_{z}$ to this decomposition, we find that $f=\partial_{z} g_{1}+\partial_{z} g_{2}$, where $\partial_{z} g_{1} \in A^{p}(\Omega)$ and $\partial_{z} g_{2} \in A^{p^{\prime}}(\Omega)^{\perp}$, the latter in view of Lemma 2.1. As for uniqueness, suppose $f_{1}+f_{2}=0$, with $f_{1} \in A^{p}(\Omega)$ and $f_{2} \in A^{p^{i}}(\Omega)^{\perp}$. We take the conjugate Cauchy transform as above

$$
\overline{\mathfrak{C}}[h](z)=\int_{\mathbb{C}} \frac{h(\zeta)}{\bar{z}-\bar{\zeta}} d A(\zeta), \quad z \in \mathbb{C},
$$

and apply it to this equality, to get

$$
\overline{\mathfrak{C}}\left[f_{1}\right](z)+\overline{\mathfrak{C}}\left[f_{2}\right](z)=0
$$

here, $\left.\overline{\mathfrak{C}}\left[f_{1}\right]\right|_{\Omega} \in H W^{1, p}(\Omega)$ and $\left.\overline{\mathfrak{C}}\left[f_{2}\right]\right|_{\Omega} \in W_{0}^{1, p}(\Omega)$, by Lemma 2.1. The uniqueness in the decomposition of the Sobolev space tells us that $\left.\overline{\mathfrak{C}}\left[f_{1}\right]\right|_{\Omega}=0$ and $\left.\overline{\mathfrak{C}}\left[f_{2}\right]\right|_{\Omega}=0$, which after an application of $\partial_{z}$ leads to $f_{1}=f_{2}=0$.

Finally, consider the implication (b) $\Rightarrow$ (d). We take a function $g \in W^{1, p}(\Omega)$, and look for a decomposition $g=g_{1}+g_{2}$, where $g_{1} \in H W^{1, p}(\Omega)$ and $g_{2} \in W_{0}^{1, p}(\Omega)$. The function $f=\partial_{z} g$ is in $L^{p}(\Omega)$, so that it has a unique decomposition $f=f_{1}+f_{2}$, where $f_{1} \in A^{p}(\Omega)$ and $f_{2} \in A^{p^{\prime}}(\Omega)^{\perp}$. Applying the conjugate Cauchy transform, we get

$$
\overline{\mathfrak{C}}[f](z)=\overline{\mathfrak{C}}\left[f_{1}\right](z)+\overline{\mathfrak{C}}\left[f_{2}\right](z)
$$


where $\left.\overline{\mathfrak{C}}\left[f_{1}\right]\right|_{\Omega} \in H W^{1, p}(\Omega)$ and $\left.\overline{\mathfrak{C}}\left[f_{2}\right]\right|_{\Omega} \in W_{0}^{1, p}(\Omega)$. The function $g-\left.\overline{\mathfrak{C}}[f]\right|_{\Omega}$ is antianalytic in $W^{1, p}(\Omega)$, and so we get the decomposition

$$
g=g-\overline{\mathfrak{C}}[f]+\overline{\mathfrak{C}}[f]=\left(g-\overline{\mathfrak{C}}[f]+\overline{\mathfrak{C}}\left[f_{1}\right](z)\right)+\overline{\mathfrak{C}}\left[f_{2}\right](z)
$$

on $\Omega$, which is of the desired type. The final item is the uniqueness. So suppose $g_{1} \in H W^{1, p}(\Omega)$ and $g_{2} \in W_{0}^{1, p}(\Omega)$ have sum $g_{1}+g_{2}=0$. Apply $\partial_{z}$ to this equality to obtain $\partial_{z} g_{1}+\partial_{z} g_{2}=0$, where $\partial_{z} g_{1} \in A^{p}(\Omega)$ and $\partial_{z} g_{2} \in \partial_{z} W_{0}^{1, p}(\Omega)=A^{p^{\prime}}(\Omega)^{\perp}$. By the uniqueness of the decomposition for $L^{p}(\Omega)$, we get $\partial_{z} g_{1}=\partial_{z} g_{2}=0$. Doing the same analysis on the conjugated equality $\bar{g}_{1}+\bar{g}_{2}=0$ leads to $\bar{\partial}_{z} g_{1}=\vec{\partial}_{z} g_{2}=0$. We conclude that both $g_{1}$ and $g_{2}$ are constant. But then $g_{2}$, being an element of $W_{0}^{1, p}(\Omega)$, must equal 0. After all, if not, then there should exist functions $G_{j} \in C_{c}^{\infty}(\Omega)$ that converge to the characteristic function $1_{\Omega}$ in the norm of $W^{1, p}(\mathbb{C})$, and then $\left.\partial_{z} G_{j}\right|_{\Omega} \rightarrow 0$ in $L^{p}(\Omega)$. But we have from Green's formula that

$$
-\int_{\Omega} z \partial_{z} G_{j}(z) d A(z)=\int_{\Omega} G_{j}(z) d A(z)
$$

the right hand side tends to the normalized area of $\Omega$ as $j \rightarrow+\infty$, whereas the left hand side tends to 0 . This contradiction shows that it is not possible to get $1_{\Omega}$ in $W_{0}^{1, p}(\Omega)$. The proof is complete.

Corollary 4.3. Let $p$ be in the interval $p_{1}<p<p_{1}^{\prime}$, where $p_{1} \approx 1.413$ and $p_{1}^{\prime} \approx 3.422$. Then the Dirichlet problem is solvable in the Sobolev class $W^{1, p}(\Omega)$ on every bounded simply connected domain $\Omega$ other than $\mathbb{C}$ itself.

Consequences for the Green solver. If $\Omega$ is a bounded domain in $\mathbb{C}$ with smooth boundary, we have a Green function $G=G_{\Omega}$, which is a function of two complex variables that for each fixed $\zeta \in \Omega$ solves the problem

$$
\begin{aligned}
\Delta_{z} G(z, \zeta) & =\delta_{\zeta}(z), \quad z \in \Omega, \\
G(z, \zeta) & =0, \quad z \in \partial \Omega,
\end{aligned}
$$

where $\delta_{\zeta}$ stands for the unit point mass at $\zeta$ and

$$
\Delta_{z}=\partial_{z} \bar{\partial}_{z}=\frac{\partial^{2}}{\partial z \partial \bar{z}}=\frac{1}{4}\left(\frac{\partial^{2}}{\partial x^{2}}+\frac{\partial^{2}}{\partial y^{2}}\right), \quad z=x+i y \in \mathbb{C}
$$

is a quarter of the ordinary Laplacian. As such, it is uniquely determined. We may also associate with the function $G$ an operator, also denoted by $G$, which is given by

$$
G[f](z)=\int_{\Omega} G(z, \zeta) f(\zeta) d A(\zeta), \quad z \in \Omega
$$


defined for appropriately summable functions $f$; we refer to it as the Green solver. It solves the boundary value problem

$$
\begin{gathered}
\Delta_{z} G[f](z)=f(z), \quad z \in \Omega, \\
G[f](z)=0, \quad z \in \partial \Omega .
\end{gathered}
$$

The regularizing properties of the operator $G$ are well-known in this setting; it lifts the degree of smoothness by two units in the scale of the Sobolev spaces. We want to define the Green solver for general bounded simply connected domains; this requires specifying our requirements on $G$ clearly in terms of the Sobolev spaces. We need the space $W^{-1, p}(\mathbb{C})$, which consists of those distributions on $\mathbb{C}$ that define continuous linear functionals on $W^{1, p^{\prime}}(\mathbb{C})$. The space of restrictions to $\Omega$ of distributions in $W^{-1, p}(\mathbb{C})$ is denoted by $W^{-1, p}(\Omega)$; it can be identified with the dual space of $W_{0}^{1, p^{\prime}}(\Omega)$. From the standard ellipticity theory for the Laplace operator $\Delta_{z}$, we have that, locally, distributions of class $W^{-1, p}$ are precisely the Laplacians of functions of class $W^{1, p}$ (see any book on elliptic partial differential equations). For bounded $\Omega$ with smooth boundary, the Green solver is characterized uniquely as the mapping $G_{\Omega}: W^{-1,2}(\Omega) \rightarrow W_{0}^{1,2}(\Omega)$ for which $\Delta G_{\Omega}$ is the identity operator on $W^{-1,2}(\Omega)$. Actually, $\Delta: W_{0}^{1,2}(\Omega) \rightarrow W^{-1,2}(\Omega)$ is invertible, and $G_{\Omega}$ is the inverse operator. As a matter of fact, this definition makes sense for general bounded domains $\Omega$. However, we are only interested in simply connected ones here.

Theorem 4.4. Let $\Omega$ be a bounded simply connected domain in $\mathbb{C}$. Then $G_{\Omega}$ defines a continuous map $W^{-1, p}(\Omega) \rightarrow W_{0}^{1, p}(\Omega)$, for all $p$ in the interval $p_{1}<p<p_{1}^{\prime}$, where $p_{1} \approx 1.413$ and $p_{1}^{\prime} \approx 3.422$.

Proof. Let $f \in W^{-1, p}(\Omega)$, and let $\tilde{f} \in W^{-1, p}(\mathbb{C})$ be a compactly supported extension of the distribution $f$ to all of $\mathbb{C}$. By the ellipticity of $\Delta$, there exists a function $g \in W^{1, p}(\mathbb{C})$ with $\Delta g=\tilde{f}$ in some open disk of large radius that contains $\bar{\Omega}$ and the support of $\tilde{f}$ compactly. By Corollary 4 , we find a (unique) function $g_{1} \in H W^{1, p}(\Omega)$ such that $g_{2}=\left.g\right|_{\Omega}-g_{1} \in W_{0}^{1, p}(\Omega)$. This function has Laplacian $\Delta g_{2}=f$, so it must be identifiable with $G[f]$; we should check that $g_{2}$ is uniquely determined by $f$. To this end, we take $f=0$, and try to prove that $g_{2}=0$. But then $\Delta g_{2}=0$, so that $g_{2} \in H W^{1, p}(\Omega)$; but we already know that $g_{2} \in W_{0}^{1, p}(\Omega)$. The uniqueness is now immediate from $H W^{1, p}(\Omega) \cap W_{0}^{1, p}(\Omega)=\{0\}$.

We should mention that Solovyov [38] has analogous applications of his (somewhat different) results on the boundedness of the Bergman projection in $L^{p}$. Corollary 4.3 is obtained for Lipschitz domains with the optimal range of ex- 
ponents $p$ ( $p$ should belong to some neighborhood of the interval $\left[\frac{4}{3}, 4\right]$ ) by Jerison and Kenig in [29]; the result extends the work of Dahlberg [19] mentioned below.

By the Sobolev imbedding theorem, the identity mapping

$$
W_{0}^{1, p^{\prime}}(\Omega) \rightarrow L^{q^{\prime}}(\Omega), \quad p^{\prime} \leq q^{\prime} \leq \frac{2 p^{\prime}}{2-p^{\prime}},
$$

is continuous provided $1<p^{\prime}<2$. Taking adjoints, then, we find that the identity mapping

$$
L^{q}(\Omega) \rightarrow W^{-1, p}(\Omega), \quad \frac{2 p}{2+p} \leq q \leq p,
$$

is continuous for $2<p<+\infty$. Combining this information with Theorem 4.4, we obtain the following result, which generalizes (albeit to a smaller range of the parameter $p$ ) the results obtained by Dahlberg [19] for Lipschitz domains to general bounded simply connected domains (where the boundary is a fairly arbitrary continuum).

Corollary 4.5. Let $\Omega$ be a bounded simply connected domain in $\mathbb{C}$. Then $G_{\Omega}$ defines a continuous map $L^{q}(\Omega) \rightarrow W_{0}^{1, p}(\Omega)$, where $1 / q=1 / p+1 / 2$, for all $p$ in the interval $2<p<p_{1}^{\prime}$, with $p_{1}^{\prime} \approx 3.422$.

Remarks on Brennan's conjecture and elliptic regularity. Let $K$ be a compact continuum in $\mathbb{C}$ which does not divide the plane, and let $\Omega$ be an open neighborhood of $K$. Suppose the function $h$ is continuous on $\Omega$ and harmonic in $\Omega \backslash K$, and that $\left.h\right|_{\Omega} \geq 0$ while $\left.h\right|_{K}=0$. The question is whether $h$ is automatically of smoothness class $W^{1, p}$ near $K$, for all $p$ in the interval $1<p<4$. This is like asking for an elliptic regularity theorem with very irregular boundary. From what we have already seen, it is clear that this amounts to a reformulation of Brennan's conjecture. Localizing to some point $z_{0} \in K$, we see that it is not the connectedness of $K$ per se that is vital for the problem, but rather a degree of "thickness" of $K$ near $z_{0}$. By elliptic regularity results for $\Delta, h$ is of smoothness class $W^{1, p}$ near $K$ if and only if $\Delta h$ is of smoothness class $W^{-1, p}$ near $K$. And $\Delta h$ is a positive distribution supported on $K$, that is, a positive Borel measure with support in $K$; it behaves like harmonic measure for some fixed point in $\mathbb{C} \backslash K$, with respect to the domain $\mathbb{C} \backslash K$. It can be shown that Brennan's conjecture is also equivalent to the statement that harmonic measure, considered as a distribution in the plane, belongs to the Sobolev space $W^{-1, p}(\mathbb{C})$, for all exponents $p$ with $1<p<4$. The classical theorem of Beurling [10, p. 53] (from his thesis, Etudes sur un problème de majoration) shows that $h$ is of Hölder class Lip $\frac{1}{2}$ near $K$, while the Sobolev imbedding theorem together with smoothness $W^{1, p}$ for $p<4$ yields a similar result. 
Harmonic measure on a continuum, generally speaking, is largest (that is, worst with respect to smoothness classes) near "tip points", so it is natural that those should be decisive for Brennan's conjecture. This should be compared with the much more precise statement of Carleson and Makarov [18]; see also [9]. We make one more remark, based on the Harnack inequality. The statement that $h$ is of smoothness class $W^{1, p}$ near $K$ means that

$$
\int_{U \backslash K}\left(\frac{h(z)}{d(z, K)}\right)^{p} d A(z)<+\infty
$$

for some neighborhood $U$ of $K$ that is (precompactly) contained in $\Omega$, where $d(z, K)$ is the Euclidean distance from $z$ to the set $K$. A much more general conjecture, due to Kraetzer [31], incorporating the conjectures of Brennan and of Carleson and Jones [17], can be expressed as the claim that

$$
\int_{U \backslash K} \frac{h(z)^{p}}{d(z, K)^{q}} d A(z)<+\infty
$$

holds provided that $0 \leq q \leq 4$ and $q^{2} / 4<p<+\infty$.

If, however, $K$ is only assumed to be compact and allowed to be disconnected, it is generally impossible to get much more than the rather trivial information that the function $h$ is of smoothness class $W^{1,2}$. For instance, if $K$ is of Cantor type, $h(z)$ can drop like $\left|z-z_{0}\right|^{\delta}$ in a small angular sector around the given point $z_{0} \in K$, for some positive constant $\delta$ that we can make as small as we like; this prevents $h$ from being in a much better Sobolev class.

An open problem. An interesting question that seems to be open is to what extent the Dirichlet problem is solvable in fractional exponent Sobolev spaces $W^{\alpha, p}$ on general bounded simply connected domains. For Lipschitz domains, the exact range of $(\alpha, p)$ was found by Jerison and Kenig [29]. The problem is of particular interest for $p=2$.

\section{Acknowledgements}

Firstly, I thank the Göran Gustafsson Foundation for providing generous financial support for the present work.

Secondly, I thank Vasilir Vasyunin for several helpful conversations in St. Petersburg and Victor Havin for mentioning the work of Solovyov; I should not forget to mention Alexander Volberg, who helped me understand Green functions for Cantor-like boundaries, and Vladimir Mazya, who supplied valuable remarks at an early stage of this work. I ought to thank Peter (Wilcox) Jones for a valuable 
comment made in Jyväskylä, and Eero Saksman for being such a good listener. Thanks are also due to Alexandru Aleman and Serguer Shimorin for help with several technical points in this manuscript.

Finally, I thank the referee for several helpful remarks.

\section{REFERENCES}

[1] R. A. Adams, Sobolev Spaces, Pure and Applied Mathematics, Vol. 65, Academic Press, New York-London, 1975.

[2] L. V. Ahlfors, Lectures on Quasiconformal Mappings, D. Van Nostrand Co., Princeton, N.J., 1966.

[3] A. Aleman, Finite codimensional invariant subspaces in Hilbert spaces of analytic functions, J. Funct. Anal. 119 (1994), 1-18.

[4] A. Aleman and A. G. Siskakis, Integration operators on Bergman spaces, Indiana Univ. Math. J. 46 (1997), 337-356.

[5] D. Békollé and A. Bonami, Inégalités à poids pour le noyau de Bergman, C. R. Acad. Sci. Paris, Sér. A-B 286 (1978), A775-A778.

[6] D. Békollé, Projections sur des espaces de fonctions holomorphes dans des domaines plans, Canad. J. Math. 38 (1986), 127-157.

[7] S. Bergman, The Kernel Function and Conformal Mapping, Second revised edition, American Mathematical Society, Providence, R.I., 1970.

[8] D. Bertilsson, Coefficient estimates for negative powers of the derivative of univalent functions, Ark. Mat. 36 (1998), 255-273.

[9] D. Bertilsson, On Brennan's conjecture in conformal mapping, Doctoral Thesis, KTH Stockholm, 1999.

[10] A. Beurling, The collected works of Arne Beurling, Vol. 1, Complex Analysis (L. Carleson, P. Malliavin, J. Neuberger and J. Wermer, eds.), Birkhäuser Boston, Boston, MA, 1989.

[11] L. Bieberbach, Ober die Koeffizienten derjenigen Potenzreihen, welche eine schlichte Abbildung des Einheitskneises vermitteln, Sitzungsber. Preuss. Akad. Wiss. 38 (1916), 940-955.

[12] A. Borichev and H. Hedenmalm, Harmonic functions of maximal growth: invertibility and cyclicity in Bergman spaces, J. Amer. Math. Soc. 10 (1997), 761-796.

[13] J. Burbea, The Bergman projection over plane regions, Ark. Mat. 18 (1980), 207-221.

[14] J. E. Brennan, The integrability of the derivative in conformal mapping, J. London Math. Soc. (2) 18 (1978), 261-272.

[15] L. Carleson, Selected Problems on Exceptional Sets, Van Nostrand Co., Princeton, N.J., 1967.

[16] L. Carleson and T. W. Gamelin, Complex Dynamics, Springer-Verlag, New York, 1993.

[17] L. Carleson and P. W. Jones, On coefficient problems for univalent functions and conformal dimension, Duke Math. J. 66 (1992), 169-206.

[18] L. Carleson and N. G. Makarov, Some results connected with Brennan's conjecture, Ark. Mat. 32 (1994), 33-62.

[19] B. E. J. Dahlberg, $L^{q}$-estimates for Green potentials in Lipschitz domains, Math. Scand. 44 (1979), 149-170.

[20] P. L. Duren, Univalent Functions, Springer-Verlag, New York, 1983.

[21] J. Feng and T. H. MacGregor, Estimates of integral means of the derivatives of univalent functions, J. Analyse Math. 29 (1976), 203-231. 
[22] T. W. Gamelin and J. B. Garnett, Distinguished homomorphisms and fiber algebras, Amer. J. Math. 92 (1970), 455-474.

[23] J. B. Gamett, Bounded Analytic Functions, Academic Press, Inc., New York-London, 1981.

[24] L. 1. Hedberg, Spectral synthesis in Sobolev spaces, and uniqueness of solutions of the Dirichlet problem, Acta Math. 147 (1981), 237-264.

[25] L. I. Hedberg and T. H. Wolff, Thin sets in nonlinear potential theory, Ann. Inst. Fourier (Grenoble) 33 (1983), 161-187.

[26] H. Hedenmalm, B. Korenblum and K. Zhu, Theory of Bergman Spaces, Springer-Verlag, New York, 2000.

[27] H. Helson and G. Szegö, A problem in prediction theory, Ann. Mat. Pura Appl. (4) 51 (1960), 107-138.

[28] R. A. Hunt, B. Muckenhoupt and R. L. Wheeden, Weighted norm inequalities for the conjugate function and Hilbert transform, Trans. Amer. Math. Soc. 176 (1973), 227-251.

[29] D. Jerison and C. E. Kenig. The inhomogeneous Dirichlet problem in Lipschitz domains, J. Funct. Anal. 130 (1995), 161-219.

[30] P. Koebe, Uber die Uniformisierung beliebiger analytischer Kurven, Nachr. Kgl. Ges. Wiss. Göttingen, Math.-Phys. Kl. (1907), 191-210.

[31] P. Kraetzer, Experimental bounds for the universal integral means spectrum of conformal maps, Complex Variables Theory Appl. 31 (1996), 305-309.

[32] N. G. Makarov, Fine structure of harmonic measure, St. Petersburg Math. J. 10 (1999), $217-$ 268.

[33] Z. Nehari, Conformal Mapping (Reprinting of the 1952 McGraw-Hill edition), Dover Publications, Inc., New York, 1975.

[34] K. Nyström, Integrability of Green potentials in fractal domains, Ark. Mat. 34 (1996), 335381.

[35] Ch. Pommerenke, Schlichte Funktionen und analytische Funktionen von beschränkter mittlerer Oszillation, Comment. Math. Helv. 52 (1977), 591-602.

[36] Ch. Pommerenke, Boundary Behaviour of Conformal Maps, Springer-Verlag, Berlin, 1992.

[37] A. A. Solov'ev, Estimates in $L^{p}$ of the integral operators that are connected with spaces of analytic and harmonic functions (Russian), Dokl. Akad. Nauk SSSR 240 (1978), 1301-1304. English translation: Soviet Math. Dokl. 19 (1978), 764-768.

[38] A. A. Solov'ev, Estimates in $L^{p}$ of integral operators connected with spaces of analytic and harmonic functions (Russian), Sibirsk. Mat. Zh. 26 (1985), 168-191, 226. English translation: Siberian Math. J. 26 (1986), 440-460.

[39] E. M. Stein, Singular Integrals and Differentiability Properties of Functions, Princeton University Press, Princeton, N.J., 1970.

[40] E. M. Stein, Harmonic Analysis: Real-Variable Methods, Orthogonality, and Oscillatory Integrals, Princeton University Press, Princeton, NJ, 1993.

[41] J. Wiegerinck, Domains with finite-dimensional Bergman space, Math. Z. 187 (1984), 559562.

\section{Håkan Hedenmalm \\ DEPARTMENT OF MATHEMATICS \\ LUND UNIVERSITY \\ BoX 118 \\ S-22100 LUND, SWEDEN \\ email: haakan@maths.Ith.se}

\author{
New address \\ DEPARTMENT OF MATHEMATICS \\ THE ROYAL. INSTTTUTE OF TECHNOLOGY \\ 10044 STOCKHOLM, SWEDEN \\ email: haakanhemath.kth.se
}

(Received November 4, 2001 and in revised form March 6, 2002) 